\title{
ImmGen at 15
}

\begin{abstract}
Nature Immunology's $20^{\text {th }}$ anniversary is a good opportunity to reminisce about the ImmGen collective endeavor - its goals, successes and horror stories - and the group's exploration of various modes of scientific publishing.
\end{abstract}

\section{The Immunological Genome Project}

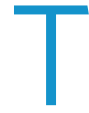
he Immunological Genome Project (ImmGen) is a collaborative group of immunology and computational biology laboratories that perform a thorough dissection of gene expression and its regulation in the immune system of the mouse. This activity first centered on mRNA expression and then expanded to microRNA (miRNA), chromatin structure, nuclear organization and protein-RNA relationships. Shared protocols, data generation and QC pipelines have yielded data that can be directly compared from $>250$ stem, lymphoid and myeloid cell types, at baseline or under challenge. The group develops and applies computational tools to decipher regulatory connections and transcriptional control. From its inception, data generated by ImmGen were meant to be a public resource, and they can be accessed through dedicated web and smartphone platforms that use interactive graphic displays that make the results intuitive to users.

\section{Basic tenets}

ImmGen has been an interesting example of consortium science ${ }^{1}$, wherein each member performs a focused exploration of their particular interest and, in doing so, contributes to a larger whole. Participation resulted in varied experiences, as reflected in collected soundbites at https://docs.google. com/spreadsheets/d/1_nNvGduRXox0sqf DSydxoLNH0eZ50W4RVMJ7hGNMZfw/ edit\#gid $=0$ Several tenets have distinguished this activity. First, in vivo veritas: only ex vivo cells are analyzed, which avoids the biases of established cell lines or cytokine-dependent primary cultures (a choice that certainly carried technical challenges). Second, to ensure data are as comparable as possible, shared pipelines are used for the genomic steps (profiling and ATAC-seq (assay for transposase-accessible chromatin using sequencing)), performed on cells that have been sorted according to rigorous standards in different labs and that are shipped to a central location. Finally, to match computational with experimental rigor, Venn diagrams were banished.

ImmGen is all mouse. Naturally, an extension to the human immune system was suggested many times but was not

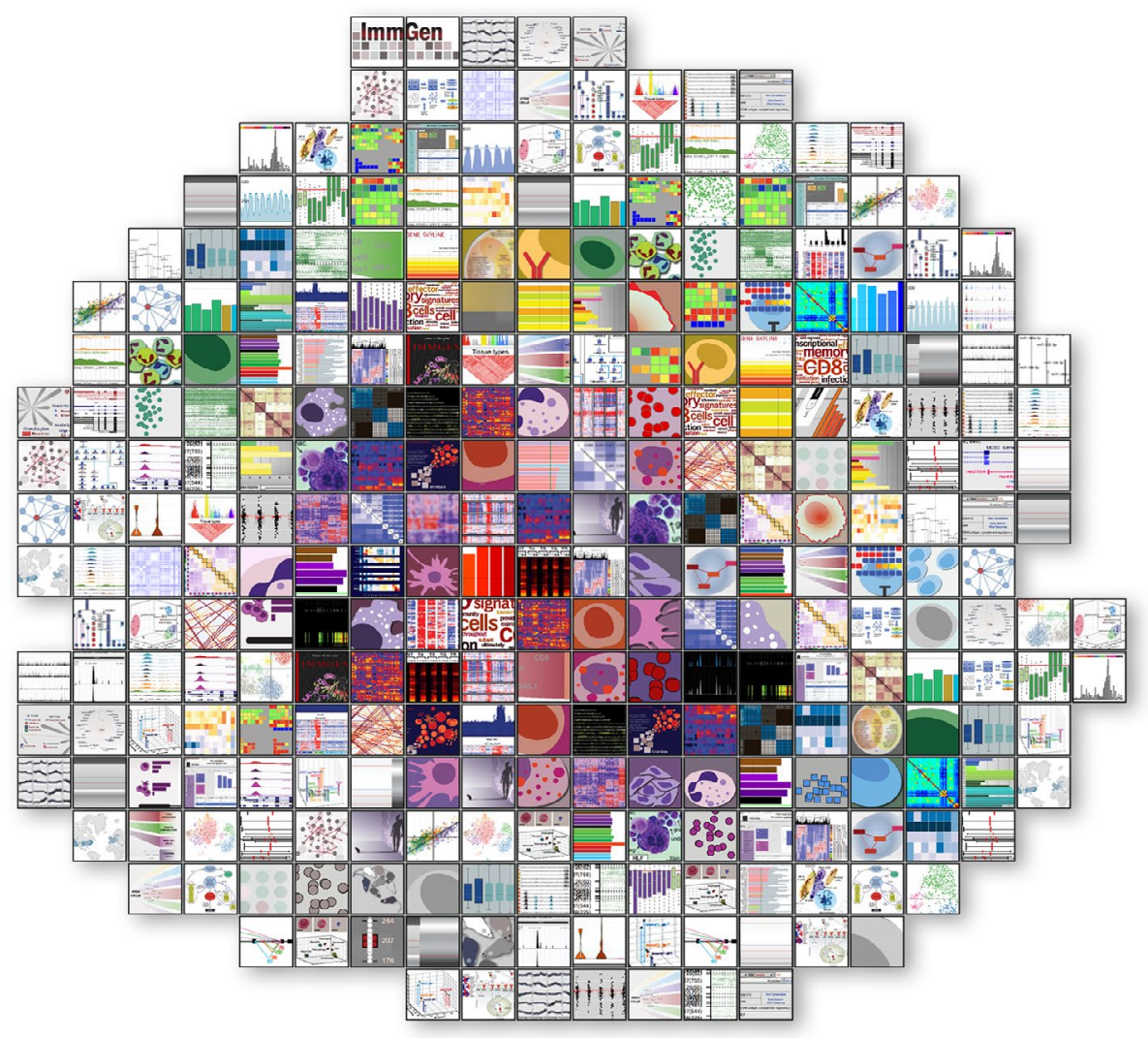

Catherine Laplace's rendition of ImmGen productions.

implemented, principally because the magnitude of the task would overwhelm a group already stretched thin, requiring new structures and logistics. On the other hand, as highlighted by ImmGen results ${ }^{2,3}$, the immune systems of mice and humans are remarkably similar in structure and regulation, when properly compared (every recent success in immunotherapy derives from mouse pilots). In addition, ImmGen results proved instrumental in opening vistas into the human immune system. While exploring dendritic cells (DCs) from mouse parenchymal or lymphoid organs, we discovered a module of genes distinguishing 'migratory DCs' that had descended to the draining lymph node from both the DCs in the tissue they came from and lymphoid-resident DCs ${ }^{4}$. DCs with precisely the same pattern of gene expression were recognizable in single-cell RNA sequencing (scRNA-seq) of human tumors, and we recently realized that the signature was in fact independent of migration and was instead triggered by uptake of cell-associated antigens and includes a strong immunoregulatory component. In addition, ImmGen data browsers do host human datasets (including topical COVID-19-related datasets), and several ImmGen members partake in the Immune Cell Atlas within the Human Cell Atlas ${ }^{5}$.

\section{Evolving technologies: what is a cell type?}

While the ultimate goals and mission have not really changed, technological advances have expanded the breadth and depth of the project (accompanied by some turnover in groups and several students and postdocs 
becoming ImmGen principal investigators). Starting from quasi-exclusive profiling of protein-coding genes, explorations expanded to include miRNAs (S.R. et al., unpublished observations), chromatin marks and structure by ATAC-seq and ChIP-seq (chromatin immunoprecipitation followed by sequencing) in the Buenrostro and Josefowicz labs ${ }^{6}$, and three dimensional nuclear architecture (HiChIP, $\mathrm{HiC}$ (high-throughput chromosome conformation capture)). Finally, ImmGen is beginning to relate mRNA and protein expression in a large proteogenomics effort with BioLegend, using DNA-tagged antibodies to combine transcriptome and surface proteome data.

ImmGen profiling started with Affymetrix arrays, which yielded tight data for a 30,000-cell input, allowing identification of cell populations at high resolution. Replacement by ULI (ultra-low-input RNA-seq) improved information in the low-expression range, and the 1,000-cell requirement opened the door to profiling of rare populations such as non-classical $\mathrm{T}$ cells or innate lymphoid cells. A third expansion came with the advent of scRNA-seq, in which some ImmGen members played a lead role. Single-cell profiling could be construed as the final step of the 'subset-splitting' that immunologists have long engaged in ( $\mathrm{B}$ and T lymphocytes, and so on). It represented a sea change in ImmGen's operational principle - which, until then, was anchored by expertly defined cell populations - as it held the promise of an unbiased and definitive atlas of cell types. But scRNA-seq did not quite yield the clarity that was hoped for. For some lineages, for example, DCs, existing populations were confirmed, and new populations emerged. But this was not the case for other cells, such as T cells, for which previously defined subsets have melted away (E. Kiner, et al., unpublished observations), and even boundaries with other lineages have become blurred. For instance, in splenocyte datasets, natural killer $(\mathrm{NK})$ and $\mathrm{CD}^{+} \mathrm{T}$ cells run together. There may be technical reasons (for example, sparsity of scRNA-seq data), but this also brings back earlier observations from population profiling: in early principal component analysis plots, NK cells and activated $\mathrm{CD}^{+} \mathrm{T}$ cells were surprisingly close, and kinetic analysis of NK cells and $\mathrm{CD}^{+} \mathrm{T}$ cells responding to viral infections by the Lanier and Goldrath labs ${ }^{7,8}$ identified shared features of these cytotoxic lymphocytes, with parallels between resting NK cells and 'central memory' $\mathrm{CD}^{+}$ $\mathrm{T}$ cells, or cytomegalovirus-memory NK cells and vesicular stomatitis virus-specific 'effector memory' CD8 ${ }^{+} \mathrm{T}$ cells (similar parallels could be made between T cells and other innate lymphoid cell populations in the Colonna $\left.\mathrm{lab}^{9}\right)$. In the overall design of the immune system, $\mathrm{NK}$ and $\mathrm{CD} 8^{+}$ $\mathrm{T}$ cells are very different actors, separated by the adaptive $\mathrm{T}$ cell receptor. This then raises the question of how to define a cell type's identity: should it be defined by its broader transcriptome or by one or a few determining genes?

\section{Success stories}

One of the key assets of ImmGen has been the deep and complementary expertise of its participants, with each of the member laboratories bringing knowledge and know-how on specific facets, lineages or cell types. This unique set has allowed the group to specialize in a range of cells, from stem cells in the Wagers and Rossi labs ${ }^{10}$, to mast cells in the Austen lab ${ }^{11}$, to stromal cells in the Turley lab ${ }^{12}$, and every lymphocyte in between. The results obtained for macrophages further illustrate this diversity. It is obvious today that tissue-resident macrophages are remarkably diverse in origin and phenotype, although this was not the case when ImmGen started. The Turley lab was initially asked to analyze all 'accessory' myeloid cells, realized that this would be a monumental task, and recruited the Merad and Randolph labs. The inclusion of macrophage populations was considered necessary but was primarily a way to improve our understanding of the DCs, which were more fashionable at that time. Research on macrophages was a backwater compared with that on DCs, and macrophages in different tissues were presumed to be functionally interchangeable. Work within ImmGen helped to rectify that erroneous conception, uncovering unexpectedly large differences between macrophages from different tissues $^{13}$. Because the consortium allowed comparison with all the other immune cell types profiled, macrophages stood out as the most diverse among the lineages. This appreciation had practical impacts: never again would it be acceptable to utilize simplistic strategies to universally identify macrophages as CD $11 b^{+}$, or DCs as the only myeloid cells expressing CD11c. This demonstration of the diversity of macrophages (and mononuclear phagocytes (MNPs) in general) was recently taken to the next level with ImmGen's MNP OpenSource program, in which many labs outside ImmGen contributed to what is an astounding collection of data ${ }^{14}$ (A. Gainullina et al., unpublished observations). Similarly, ImmGen studies in the Monach lab also shone a new light on neutrophil diversity during activation ${ }^{15}$.

The analysis of long-lived tissue-resident mast cells (MCs) ${ }^{11}$ also illustrated the power of complementarity. Both MCs and basophils express the IgE receptor Fcerla, although they predate IgE by hundreds of millions of years. Basophils emerge from the bone marrow as mature effectors with a short lifespan, whereas MCs only mature within tissues. ImmGen provided the unique opportunity to place these ancient cells within the context of the modern mammalian immune system. MCs proved to be incredibly distinct, forming an independent cluster separate from lymphoid and other myeloid cells. By contrast, basophils clustered with eosinophils and neutrophils and had far more in common with other circulating granulocytes than with MCs. We also identified a core connective tissue MC signature distinct from mucosal mast cells.

Finally, areas too big for any of us to tackle alone included the full differentiation cascade of B cells, undertaken in the Hardy and Nutt labs, and the large galaxy of $\mathrm{T}$ lymphocytes, which spans somatically adaptive and innate-like moieties. It took the combined expertise of the Kang $\left(\gamma \delta \mathrm{T} \mathrm{cells}^{16}\right)$, Brenner and Kronenberg (non-classical innate-like $\alpha \beta$ T cells ${ }^{17}$ ), CBDM (differentiation ${ }^{18}$ ) and Goldrath and Dustin (activation, effector and memory ${ }^{8}$ ) laboratories to stitch together what is certainly the broadest survey of transcription and the chromatin accessibility landscape anywhere. Whereas effector $\mathrm{T}$ subtypes share gene programs to execute their function, distinctions emerged in how functional specialization is achieved, from preprograming of innate-like effector subtypes to becoming adaptive effectors post-antigen experience.

\section{Mistakes and horror stories}

Naturally, the group had ups and downs, some mistakes and dead-ends. At an unusually tense ImmGen workshop several years ago, the core team was taken to task for its persistent failure to develop a robust RNA-seq protocol compatible with low cell numbers. Luckily, the Broad Technology Labs devised a fabulous implementation of SmartSeq2 for low-input profiling that sidestepped RNA purification, and it has been ImmGen's workhorse ever since. Smiles returned. Some cell populations had to be pulled from the website because of contamination issues that had not been initially realized (in some cases, because surface markers for sorting were not as specific as had been thought). Proteome profiling also proved challenging. 
In spite of the best intentions and efforts, collaborations with the systematic mouse mutagenesis programs (M. Malissen and Phenomin, Knockout Mouse Project (KOMP)) never yielded very striking results, partly because gene choices and timelines proved hard to articulate, and because many single knockouts turned out to have little impact on the transcriptomes of $\mathrm{CD}^{+}$ $\mathrm{T}$ cells or macrophages, indicating a strong resilience of the regulatory network.

\section{Extracting the "substantifique moelle"}

The 15th century writer Rabelais coined the metaphor "substantifique moelle", advocating for meditation on texts to extract deep meaning and knowledge, as one would extract marrow from bone - or as computational analysis is able to extract meaning from Big Data. As reflected by some of its founding members (Koller, Collins and Regev), ImmGen always aimed to go beyond mere cataloging and used computational mining to exploit the data for implications of regulatory connections within the immune system. Tools for network inference were being developed in the early 2000s, and we set out to reconstruct the regulatory network of the mouse immune system. The data were huge and dynamic - new cell types were added continuously, forcing recomputation every few months. A few thousand genes capture the dynamics of most systems, but, for the entire immune system, even 8,000 genes seemed small; there were long debates on the depth of clustering that should be applied across several hundred samples, representing $>300$ cell types (a compromise was reached on two levels of clustering, coarse or fine). The next step entailed developing a novel algorithm that exploited stepwise transitions in the differentiation cascades to infer the regulatory transcription factors ${ }^{19}$. This type of approach was carried further through several projects $^{6,20-22}$ in the Shay and Mostafavi labs. Most recently, we exploited the potential of deep neural networks to learn and 'understand' highly complex and non-linear relationships in large datasets. A trained deep neural network can accurately predict, from DNA sequence alone, the activity of enhancers across the whole immune system and the transcription factors that mediate this activity ${ }^{3}$. In an overnight run, the machine rediscovers 30 years of hard-won immunogenetics. Humbling!

\section{Value as a resource}

One of the 'wow moments' came when we started monitoring traffic to the website and realized that $\sim 50$ visitors quietly consulted the site every day, whereas we had expected one or two. Someone was seeing value in the effort. This number has now grown and has stabilized at 250 independent visits per weekday, and the smartphone app also has widespread use ${ }^{23}$. The ImmGen data browsers are different from the portals of many consortia. They do not aim to serve raw datasets (repositories like the Gene Expression Omnibus did this far more professionally), but are designed to answer the diversity of "Show me..." questions that an immunologist might raise, and they aim for consultation rather than downloads, and graphic visualization rather than tables. Although the Skyline expression histograms account for half the traffic, almost all other tools are queried $>100$ times per month. To be fair, ImmGen data browsers are an idiosyncratic lot with respect to design and architecture, contributed by different software developers over time. Some are a bit quirky, and interconnections could be improved. David Laidlaw, who helped launch ImmGen visualizations, argued for a lightweight, flexible and evolving assemblage, rather than a large architected machine with industrial-strength software engineering. But ImmGen browsers are robust: the original Skyline developed 15 years ago by the Park-Seguritan-Hyatt trio is still running strong.

\section{Nature Immunology}

Fittingly for this anniversary issue, the special relationship between Nature Immunology (NI) and ImmGen was instrumental to the latter's success. The Berlin Accord (really an impromptu discussion with a senior NI editor at a poster session) acknowledged that data-rich and descriptive ImmGen reports were inherently worthwhile, and could be published without the "driving hypothesis", "mechanistic insight" and knockout follow-ups requested by Reviewer 3. However, there was a stipulation that the landscape information be broad and integrative, and it had to generate truly novel insights from the cell studies, not mere gene lists. ImmGen reports published in refs. ${ }^{7-9,11-13,16-19,21}$ met these criteria, and the agreement gave ImmGen the freedom of mind to pursue its endeavors for true resource building. But, contrary to rumors, ImmGen had no free pass at NI.

\section{When is ImmGen finished?}

What lies ahead for ImmGen? Answers to this question are exciting and tantalizing. The overall vision could be stated as providing a resource that details the expression of every gene and protein, the regulatory elements (enhancers and nuclear structures, transcription factors and regulatory RNAs) that control their expression, and how this network is brought to bear for organismal homeostasis and immune responses to challenges.

On the way to this elusive holy grail, equally ambitious stepping stones may be to fully define cell types (whether as discrete cell types or as continua, which may require a new vocabulary), to generate a developmental atlas of immune cells across tissues and time, to generate a comprehensive chart of all cis-regulatory elements that our field can adopt as a roadmap, and to link transcriptomes and proteomes. These monumental challenges will also require us to harness the all-encompassing power of machine learning and, from these foundational questions, to ask whether the blueprint provides robust nodes that can be utilized to bolster or repair fragilities and blind spots in immunity.

Immunological Genome Project

Stephanie Vargas Aguilar', Oscar Aguilar², Rhys Allan ${ }^{3}$, El Ad David Amir ${ }^{4}$, Veronique Angeli ${ }^{5}$, Maxim N. Artyomov ${ }^{6}$, Natasha Asinovski ${ }^{7}$, Jilian Astarita ${ }^{8}$, K. Frank Austen ${ }^{9}$, Geetika Bajpai ${ }^{10}$, Nora Barrett ${ }^{9}$, Alev Baysoy ${ }^{7}$, Christophe Benoist ${ }^{7 凶}$, Angelique Bellemare-Pelletier ${ }^{8}$, Brad Berg', Adam Best ${ }^{12}$, Natalie Bezman ${ }^{2}$, David Blair ${ }^{13}$, Julie M. Blander ${ }^{4}$, Milena Bogunovic ${ }^{4}$, Patrick Brennan ${ }^{14}$, Michael Brenner ${ }^{14}$, Brian Brown ${ }^{15}$, Matthew Buechler ${ }^{8}$, Jason Buenrostro ${ }^{16}$, Maria Acebes Casanova ${ }^{4}$, Kyunghee $\mathrm{Choi}^{6}$, Andrew $\mathrm{Chow}^{4}$, Aleksey Chudnovskiy ${ }^{4}$, Daniela Cipoletta7, Nadia Cohen ${ }^{14}$, James J. Collins ${ }^{17}$, Marco Colonna ${ }^{6}$, Alison Cook ${ }^{7}$, James Costello ${ }^{17}$, Viviana Cremasco ${ }^{8}$, Ty Crowl ${ }^{12}$, Karine Crozat ${ }^{1}$, Richard Cruse7, June D'Angelo6, Marc Dalod', Scott Davis ${ }^{7}$, Cagatay Demiralp ${ }^{11}$, Tianda Deng ${ }^{12}$, Jigar V. Desai ${ }^{18}$, Fiona Desland ${ }^{4}$, Maxime Dhainaut ${ }^{15}$, Jiarui Ding ${ }^{19}$, Andrew Doedens ${ }^{12}$, Claudia Dominguez ${ }^{8}$, Graeme Doran 7 , Regine Dress ${ }^{20}$, Michael Dustin ${ }^{13}$, Daniel Dwyer ${ }^{9}$, Ivan Dzhagalov' ${ }^{21}$, Kutlu Elpek ${ }^{8}$, Ayla Ergun7, Jeff Ericson ${ }^{7}$, Eunice Esomonu ${ }^{7}$, Keke Fairfax ${ }^{6}$, Anne Fletcher ${ }^{8}$, Michela Frascoli ${ }^{22}$, Anja Fuchs ${ }^{6}$, Anastasiia Gainullina ${ }^{6}$, Shani Gal-Oz ${ }^{23}$, Michael Gallagher ${ }^{22}$, Emmanuel Gautier ${ }^{24}$, Roi Gazit ${ }^{25}$, Sophie Gibbings ${ }^{26}$, Matthieu Giraud ${ }^{7}$, Florent Ginhoux ${ }^{20}$, Ananda Goldrath ${ }^{12}$, Dagmar Gotthardt'², Daniel Gray?, Melanie Greter ${ }^{4}$, Ricardo Grieshaber-Bouyer ${ }^{27}$, Martin Guilliams ${ }^{28}$, Sara Haidermota ${ }^{7}$, Randy Hardy ${ }^{29}$, Daigo Hashimoto ${ }^{4}$, Julie Helft ${ }^{4}$, Deborah Hendricks ${ }^{2}$, Tracy Heng? Jonathan Hill7, Gordon Hyatt ${ }^{7}$, Juliana Idoyaga ${ }^{4}$, 
Claudia Jakubzick ${ }^{26}$, Jessica Jarjoura ${ }^{2}$, Daniel Jepson 7 , Baosen Jia ${ }^{4}$, Radu Jianu", Tim Johanson ${ }^{3}$, Stefan Jordan ${ }^{4}$, Vladimir Jojic ${ }^{30}$, Stefan Jordan ${ }^{4}$, Yosuke Kamimura ${ }^{2}$, Veronica Kana ${ }^{4}$, Joonsoo Kang ${ }^{22}$, Varun Kapoor ${ }^{8}$, Ephriam Kenigsberg ${ }^{4}$, Andrew Kent ${ }^{4}$, Charles Kim², Edy Kim ${ }^{14}$, Francis Kim ${ }^{16}$, Joel Kim ${ }^{4}$, Kiwook Kim ${ }^{6}$, Evgeny Kiner ${ }^{7}$, Jamie Knell'12, Daphne Koller ${ }^{30}$, Larry Kozinn?, Karen Krchma ${ }^{6}$, Taras Kreslavsky Mitchell Kronenberg ${ }^{31}$, Wing-Hong Kwan ${ }^{4}$, David Laidlaw"11, Viola Lam², Lewis Lanier², Catherine Laplace ${ }^{7}$, Caleb Lareau ${ }^{16}$, Yonit Lavin ${ }^{4}$, Kory J. Lavine ${ }^{10}$, Andrew Leader ${ }^{4}$, Marylene Leboeuf ${ }^{4}$, Jacob Lee ${ }^{6}$, Jisu Lee ${ }^{22}$, Bo Li ${ }^{19}$, $\mathrm{Hu} \mathrm{Li}{ }^{17}$, Yuesheng Li ${ }^{29}$, Michail S. Lionakis ${ }^{18}$, Herve Luche ${ }^{32}$, Lydia Lynch ${ }^{14}$, Assaf Magen ${ }^{4}$, Barbara Maier ${ }^{4}$, Deepali Malhotra ${ }^{8}$, Nidhi Malhotra ${ }^{22}$, Marie Malissen ${ }^{32}$, Alexandra Maslova ${ }^{33}$, Diane Mathis ${ }^{7}$, Adelle McFarland ${ }^{6}$, Miriam Merad ${ }^{4}$, Etienne Meunier ${ }^{33}$, Jennifer Miller ${ }^{4}$, Justin Milner ${ }^{12}$, Michael Mingueneau', Gundula Min-Oo ${ }^{2}$, Paul Monach ${ }^{27}$, Devapregasan Moodley ${ }^{7}$, Arthur Mortha ${ }^{4}$, Maelig Morvan ${ }^{2}$, Sara Mostafavi ${ }^{33}$, Soren Muller ${ }^{8}$, Christoph Muus $^{19}$, Tsukasa Nabekura², Tata Nageswara Rao ${ }^{16}$, Vipin Narang ${ }^{20}$, Kavitha Narayan ${ }^{22}$, Hadas Ner-Gaon ${ }^{23}$, Quyhn Nguyen ${ }^{12}$, Peter A. Nigrovic ${ }^{27}$, German Novakovsky ${ }^{33}$, Stephan Nutt ${ }^{3}$, Kayla Omilusik ${ }^{12}$, Adriana Ortiz-Lopez ${ }^{7}$, Helena Paidassi ${ }^{34}$, Henry Paik ${ }^{7}$, Michio Painter ${ }^{7}$, Mallory Paynich ${ }^{31}$, Vincent Peng ${ }^{6}$, Marc Potempa ${ }^{2}$,

Rachana Pradhan ${ }^{8}$, Jeremy Price ${ }^{4}$, Yilin Qi ${ }^{22}$, Yiqing $\mathrm{Qi}^{7}$, Sara Quon ${ }^{12}$, Ricardo Ramirez ${ }^{7}$, Deepshika Ramanan ${ }^{7}$, Gwendalyn Randolph ${ }^{6}$, Aviv Regev ${ }^{19}$, Andrew Rhoads ${ }^{7}$,

Michelle Robinette ${ }^{6}$, Samuel Rose ${ }^{15}$,

Derrick Rossi ${ }^{25}$, Katie Rothamel ${ }^{7}$

Ravi Sachidanandam ${ }^{4}$, Priyanka Sathe ${ }^{4}$ Charlotte Scott ${ }^{28}$, Kumba Seddu ${ }^{7}$, Peter $\mathrm{See}^{20}$, Alexey Sergushichev ${ }^{6}$, Laura Shaw ${ }^{12}$, Tal Shay ${ }^{23}$, Avishai Shemesh ${ }^{2}$, Susan Shinton ${ }^{29}$, Justin Shyer ${ }^{8}$, Michael Sieweke ${ }^{1}$, Chris Smillie ${ }^{19}$, Lotte Spel ${ }^{8}$, Nick Spidale ${ }^{22}$, Giuseppina Stifano ${ }^{27}$, Ayshwarya Subramanian ${ }^{19}$, Joseph Sun ${ }^{2}$, Katelyn Sylvia' ${ }^{22}$, Julie Tellier ${ }^{3}$, Sébastien This ${ }^{34}$, Elena Tomasello', Helena Todorov ${ }^{28}$, Shannon Turley ${ }^{8}$, Brinda Vijaykumar ${ }^{7}$, Amy Wagers ${ }^{16}$, Ei Wakamatsu ${ }^{7}$, Chendi Wang ${ }^{33}$, Peter L. Wang ${ }^{6}$, Aleksandra Wroblewska ${ }^{15}$,
Jun $\mathrm{Wu}^{6}$, Edward Yang ${ }^{12}$, Liang Yang ${ }^{7}$, Aldrin Yim ${ }^{6}$, Lim Sheau Yng ${ }^{5}$, Hideyuki Yoshida ${ }^{7}$, Bingfei $\mathrm{Yu}^{12}$, Yan Zhou ${ }^{29}$, Yanan Zhu ${ }^{2}$ and Caroline Ziemkiewicz"

${ }^{1}$ French National Center for Scientific Research, Centre d'Immunologie de Marseille Luminy, Marseille, France. ${ }^{2}$ Department of Microbiology \& Immunology, University of California, San Francisco, San Francisco, CA, USA. ${ }^{3}$ The Walter and Eliza Hall Institute of Medical Research, Parkville, Victoria, Australia. ${ }^{4}$ Immunology Institute and Tisch Cancer Institute, Icahn School of Medicine at Mount Sinai, New York, NY, USA. ${ }^{5}$ Centre for Life Sciences, National University of Singapore, Singapore, Singapore. ${ }^{6}$ Department of Pathology and Immunology, Washington University School of Medicine, St. Louis, MO, USA. 'Department of Immunology, Harvard Medical School, Boston, USA. ${ }^{8}$ Department of Cancer Immunology, Genentech, South San Francisco, CA, USA. ${ }^{9}$ Division of Rheumatology, Immunology and Allergy, Brigham and Women's Hospital, Boston, MA, USA. ${ }^{10}$ Center for Cardiovascular Research, Division of Cardiology, Department of Medicine, Washington University School of Medicine, Saint Louis, MO, USA. ${ }^{11}$ Brown Computer Science, Brown University, Providence, RI, USA. ${ }^{12}$ Division of Biological Sciences, University of California, San Diego, La Jolla, CA, USA. ${ }^{13}$ Kennedy Institute of Rheumatology at the University of Oxford, Oxford, UK. ${ }^{14}$ Division of Rheumatology, Inflammation and Immunity, Brigham and Women's Hospital and Harvard Medical School, Boston, MA, USA. ${ }^{15}$ Icahn School of Medicine at Mount Sinai, New York, NY, USA. ${ }^{16}$ Department of Stem Cell and Regenerative Biology, Harvard University, Cambridge, MA, USA. ${ }^{17}$ Department of Biomedical Engineering and Center for Synthetic Biology, Boston University, Boston, MA, USA. ${ }^{18}$ National Institute of Allergy and Infectious Diseases, National Institutes of Health, Bethesda, MD, USA. ${ }^{19}$ Broad Institute of Massachusetts Institute of Technology and Harvard, Cambridge, MA, USA. ${ }^{20}$ Singapore Immunology Network, Agency for Science, Technology and Research, Singapore, Singapore. ${ }^{21}$ Institute of Microbiology and Immunology, National Yang-Ming University, Taipei, Taiwan. ${ }^{22}$ Department of Pathology, University of Massachusetts Medical School, Worcester, MA, USA. ${ }^{23}$ Department of Life Sciences, Ben-Gurion University of the Negev, Be'er Sheva, Israel. ${ }^{24}$ Faculty of Medicine, National Institute for Health and Medical Research, Paris, France. ${ }^{25}$ Immune Disease Institute, Program in Cellular and Molecular Medicine, Children's Hospital Boston, Boston, MA, USA. ${ }^{26}$ Department of
Pediatrics, National Jewish Health, Denver, CO, USA. ${ }^{27}$ Department of Medicine, Boston University, Boston, MA, USA. ${ }^{28}$ Laboratory of Immunoregulation, Inflammation Research Centre, VIB Ghent University, Ghent, Belgium. ${ }^{29}$ Fox Chase Cancer Center, Philadelphia, PA, USA. ${ }^{30}$ Computer Science Department, Stanford University, Stanford, CA, USA. ${ }^{31}$ La Jolla Institute for Immunology, La Jolla, CA, USA. ${ }^{32}$ Centre d'Immunologie de Marseille-Luminy, INSERM, CNRS, Marseille, France. ${ }^{33}$ Department of Statistics, University of British Columbia, Vancouver, BC, Canada. ${ }^{34}$ International Center for Research in Infectious Disease, University of Lyon, Lyon, France.

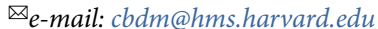

Published online: 23 June 2020

https://doi.org/10.1038/s41590-020-0687-4

References

1. Benoist, C., Lanier, L., Merad, M. \& Mathis, D. Nat. Rev. Immunol. 12, 734-740 (2012).

2. Shay, T. et al. Proc. Natl Acad. Sci. USA 110, 2946-2951 (2013).

3. Maslova, A. et al. Preprint at bioRxiv https://doi.org/ 10.1101/2019.12.21.885814 (2019).

Miller, J. C. et al. Nat. Immunol. 13, 888-899 (2012).

5. Regev, A. et al. Elife 6, e27041 (2017).

6. Yoshida, H. et al. Cell 176, 897-912.e20 (2019).

7. Bezman, N. A. et al. Nat. Immunol. 13, 1000-1009 (2012).

8. Best, J. A. et al. Nat. Immunol. 14, 404-412 (2013).

9. Robinette, M. L. et al. Nat. Immunol. 16, 306-317 (2015).

10. Gazit, R. et al. Stem Cell Rep. 1, 266-280 (2013).

1. Dwyer, D. F. et al. Nat. Immunol. 17, 878-887 (2016).

12. Malhotra, D. et al. Nat. Immunol. 13, 499-510 (2012).

13. Gautier, E. L. et al. Nat. Immunol. 13, 1118-1128 (2012).

14. ImmGen Consortium. Nat. Immunol. 17, 741 (2016).

15. Ericson, J. A. et al. PLoS ONE 9, el08553 (2014).

16. Narayan, K. et al. Nat. Immunol. 13, 511-518 (2012).

17. Cohen, N. R. et al. Nat. Immunol. 14, 90-99 (2013).

18. Mingueneau, M. et al. Nat. Immunol. 14, 619-632 (2013).

19. Jojic, V. et al. Nat. Immunol. 14, 633-643 (2013).

20. Mostafavi, S. et al. Cell 164, 564-578 (2016).

1. Gal-Oz, S. T. et al. Nat. Commun. 10, 4295 (2019).

22. Mostafavi, S. et al. J. Immunol. 193, 4485-4496 (2014).

23. Ericson, J. A., Laplace, C. \& Benoist, C. ImmGen mobile phone application (2013).

\section{Acknowledgements}

ImmGen was made possible by its sponsors and supporters, foremost the NIH NIAID, whose grant (R24-AI072073) unflinchingly supported the enterprise, but also by companies in the immunogenomics arena (eBioscience, BioLegend and Qiagen) who understood the goals and helped with collaborative participation in experimental design and discounted reagents. We thank the profiling facilities (Expression Analysis, Broad BTL and Genomics Platforms) for their professionalism and dedication in data generation.

Competing interests

The authors declare no competing interests. 